\title{
The Use of Hypnosis for the Treatment of Nocturnal Enuresis: Myth or Reality?
}

\author{
Anna Maria Della Grotta, $M D^{1}$ and Alessandro Boscarelli, $M D^{2^{*}}$ \\ ${ }^{1}$ Hypno-Psychotherapist, Clinical Center of the Italian Society of Hypnosis, Rome, Italy \\ ${ }^{2}$ Department of Pediatric Surgery and Urology, Institute for Maternal and Child Health - IRCCS \\ "Burlo Garofolo", Trieste, Italy
}

*Corresponding author: Alessandro Boscarelli, MD, Department of Pediatric Surgery and Urology, Institute for Maternal and Child Health - IRCCS "Burlo Garofolo" via dell'Istria, 65/1, 34137 Trieste (TS), Italy, Tel: 0039-040-3785372, Fax: 0039040-3785537

\begin{abstract}
Nocturnal enuresis is a common condition which may be cause of considerable psychological distress especially in children. Despite poor evidence across the literature, hypnosis could have an important role as first-line treatment option for primary monosymptomatic nocturnal enuresis.
\end{abstract}

\section{Keywords}

Hypnosis, Treatment, Nocturnal enuresis

\section{Introduction}

Nocturnal enuresis, also called bed-wetting or night-time incontinence, is a common condition which may be cause of considerable psychological distress especially in children. Nocturnal enuresis refers to the involuntary loss of urine during sleep occurring at least twice a week in patients older than 5 years of age and lasting for 3 months minimum. Nocturnal enuresis can be classified as primary or secondary. Primary nocturnal enuresis occurs in patients who have never achieved a satisfactory period of dryness, while secondary nocturnal enuresis has an onset after a period of dryness of at least 6 months [1]. Nocturnal enuresis can be also classified as monosymptomatic or non-monosymptomatic. In particular, primary monosymptomatic nocturnal enuresis (PMNE) has been defined by the International Children's Continence Society as pathological bed-wetting with at least one episode per month in patients older than 5 years of age [2], without any other lower urinary tract symptoms and no history of bladder dysfunction. PMNE is a multifactorial disease with an important genetic component, and it is influenced by the bladder control mechanisms of the central nervous system [1,3]. Even if PMNE is widely considered a self-limiting disease, it has been estimated that approximately $20-30 \%$ of patients with PMNE may have at least one behavioral, psychological or psychiatric disorder [1,4]. Management options for PMNE include behavioral modifications, psychological support, and pharmacologic therapy. Remarkably, a non-pharmacological approach is recommended as first-line treatment option because of a proven long-term beneficial effect [4]. To date, hypnosis is still underutilized for the treatment of nocturnal enuresis due to a weakness in the literature evidence. Conversely, hypnosis demonstrated to be more powerful than placebo, beneficial in the treatment of some conditions (e.g., migraine headache, irritable bowel syndrome, and anxiety), and highly effective in reducing the consumption of medications [5].

In conclusion, we believe that hypnosis could be taken into consideration as a treatment option in patients suffering from PMNE. Thus, further studies are strongly encouraged to better evaluate the precise role of hypnosis for the treatment of nocturnal enuresis.

\section{Submission Declaration}

This work has not been published previously and is 
not under consideration for publication elsewhere. It has been approved by all authors. All the authors attest that they meet the ICMJE criteria for authorship.

\section{Conflicts of Interest}

The authors have no conflicts of interest related to this article to declare.

\section{Role of the Funding Source}

This article did not receive any specific grant from funding agencies in the public, commercial, or not-forprofit sectors.

\section{References}

1. Gomez Rincon M, Leslie SW, Lotfollahzadeh S (2021) Nocturnal Enuresis. In: StatPearls [Internet]. StatPearls Publishing, Treasure Island (FL).
2. Austin PF, Bauer SB, Bower W, Chase J, Franco I, et al. (2016) The standardization of terminology of lower urinary tract function in children and adolescents: Update report from the standardization committee of the International Children's Continence Society. Neurourol Urodyn 35: 471481.

3. Fagundes SN, Lebl AS, Azevedo Soster L, Sousa E Silva GJ, Silvares EF, et al. (2017) Monosymptomatic nocturnal enuresis in pediatric patients: Multidisciplinary assessment and effects of therapeutic intervention. Pediatr Nephrol 32: 843-851.

4. Hamed SA, Fawzy M, Hamed EA (2020) Behavioral problems in children with primary monosymptomatic nocturnal enuresis. Compr Psychiatry 104: 152208.

5. Kittle J, Spiegel D (2021) Hypnosis: The most effective treatment you have yet to prescribe. Am J Med 134: 304305. 